Nervenarzt $2012 \cdot 83: 705-713$ DOI 10.1007/s00115-011-3438-9

Online publiziert: 21. März 2012

(c) Springer-Verlag 2012

C.M. Lill' ${ }^{1,2} \cdot$ F. Zipp ${ }^{1}$

${ }^{1}$ Klinik und Poliklinik für Neurologie, Johannes-Gutenberg-Universität, Mainz

${ }^{2}$ Abt. Genomik der Vertebraten, Max-Planck-Institut für molekulare Genetik, Arbeitsgruppe Neuropsychiatrische Genetik, Berlin

\title{
Genetisches Profil der Multiplen Sklerose: Risikogene und die "dunkle Materie“
}

troffener Patienten (zur Übersicht s. [6, 27]). Etwa $20 \%$ der Patienten aus Nordeuropa zeigen eine positive Familienanamnese bei einer allgemeinen Krankheitsprävalenz in der Population von 0,1$0,3 \%$. Populationsbasierte Familien- und Zwillingsstudien haben gezeigt, dass sich das Erkrankungsrisiko mit zunehmender Anzahl betroffener Familienmitglieder und zunehmendem Verwandtschaftsgrad ebenfalls erhöht: So haben ein Geschwister bzw. ein dizygoter Zwilling eines MS-Patienten ein etwa 5\%iges Lebenszeitrisiko an MS zu erkranken, während ein monozygoter Zwilling ein etwa 25\%iges Risiko aufweist. Die familiäre Aggregation scheint deutlich durch die genetische Komponente bedingt zu sein, und kulturelle oder Umweltkomponenten in der Familie scheinen hier nur einen geringen Einfluss zu haben. Dies zeigt eine Reihe von Studien: So hat etwa bei adoptierten MS-Patienten die adoptierende Familie ein Risiko für MS, das dem der normalen Bevölkerung entspricht, während die Ursprungsfamilie ein erhöhtes Risiko aufweist. Des Weiteren ist das Risiko von Halbgeschwistern ungefähr halb so groß wie das von vollen Geschwistern, unabhängig davon, ob sie getrennt oder zusammen aufgewachsen sind, und es besteht kein erhöhtes Risiko für die genetisch nichtverwandten Stiefgeschwister. Es kann daraus jedoch nicht abgeleitet werden, dass Umweltfaktoren keine Rolle spielen. Vielmehr scheinen Umweltfaktoren eher auf Populationsebene einen Einfluss zu haben, aber nur einen geringen oder keinen Einfluss innerhalb von Familien [27].

Trotz der Evidenzen für den genetischen Einfluss auf die Erkrankungswahrscheinlichkeit gibt es, anders als bei Erkrankungen wie etwa der Alzheimeroder Parkinson-Erkrankung, bei denen 5-10\% der Patienten autosomal-dominante oder -rezessive Mutationen ausweisen (zur Übersicht s. [18]), keine Hinweise auf eine nach Mendel vererbte Form der Erkrankung. Die meisten MS-Familien haben nicht mehr als 2 oder 3 Erkrankte, ohne dass ein klares Vererbungsmuster erkennbar wäre. Nur in Einzelfällen sind Familien mit mehr Erkrankten beschrieben worden (z. B. $[8,13])$. Hier gab es jedoch keine Kopplung zu chromosomalen Regionen, die auf eine hochpenetrante $\mathrm{Mu}$ tation hinweisen könnte. Vielmehr wird angenommen, dass sowohl in familiären als auch in "sporadischen“ Fällen genetische Varianten, die auch in der gesunden Bevölkerung vorkommen können, in einer Vielzahl von Genorten („Loci“) eine Prädisposition zur Entstehung von MS bewirken und in der Interaktion mit weiteren intrinsischen sowie Umweltfaktoren zu einer Erkrankung führen können. Die Erblichkeit in Populationen europäischen Ursprungs, d. h. der Anteil phänotypischer Variation in der Bevölkerung, der durch vererbbare Faktoren bedingt wird, wird auf etwa $40 \%$ geschätzt [26].

\section{Genetische Assoziationsstudien}

Genetische Assoziationsstudien untersuchen einen statistischen Zusammen- kungen ist auch MS charakterisiert durch eine erhöhte Inzidenz in Verwandten be- 
Patienten $(n=25)$

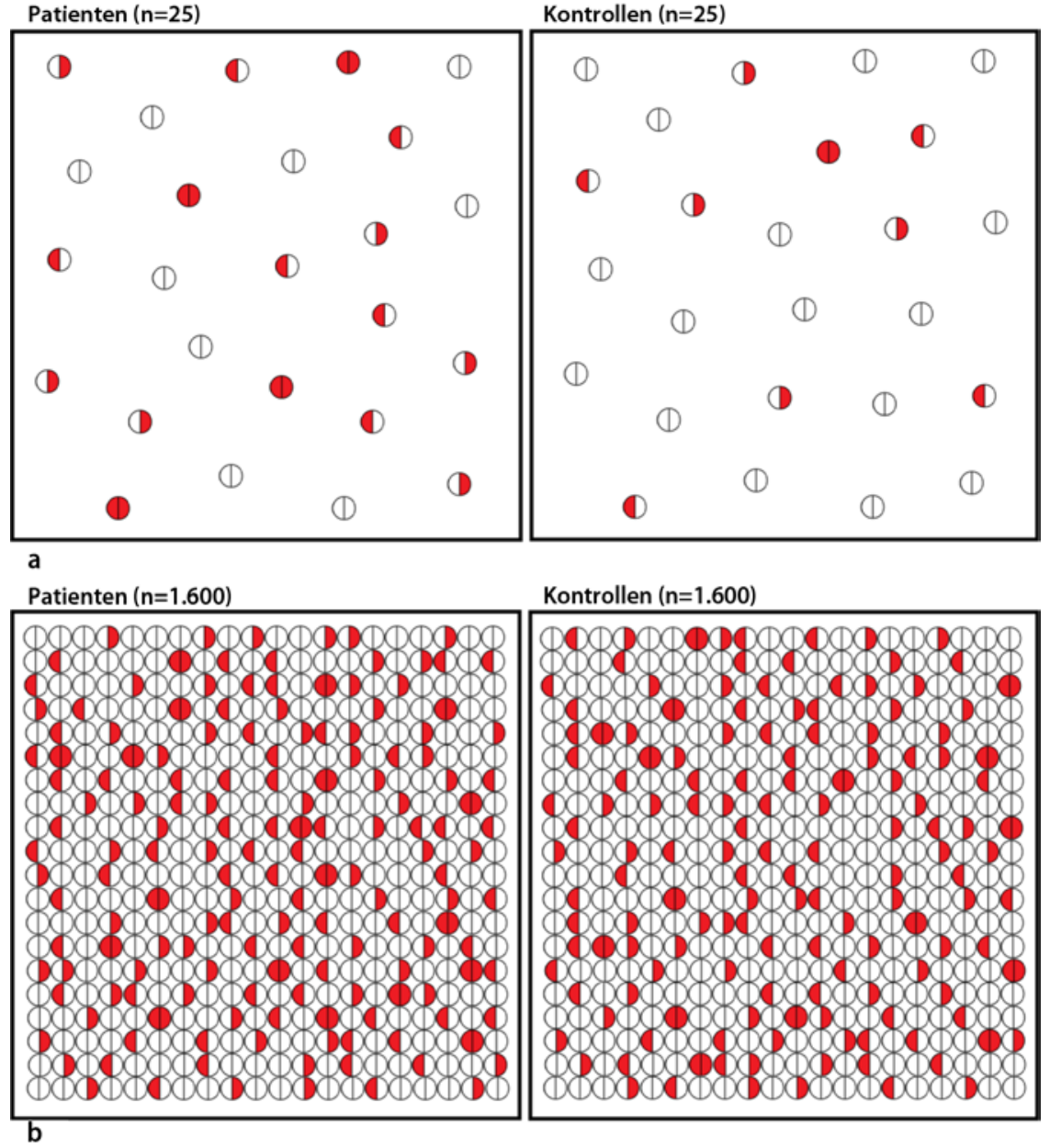

Abb. $1 \Delta$ Schematische Darstellung der Verteilung einer genetischen Risikovariante in einer Patientenund Kontrollstichprobe. Jede Person ist durch einen Kreis dargestellt und besitzt eine mütterliche und ein väterliche Kopie des Genoms. Trägt sie an der Stelle der genetischen Variante zwei Referenzallele, ist sie homozygot für das Referenzallel (weiße Kreise), trägt sie zwei Risikoallele, ist sie homozygot für das Risikoallel (rote Kreise); weist sie ein Referenzallel und ein Risikoallel auf, ist sie heterozygot für die Variante (weiß-rote Kreise). In • Abb. 1a ist die Verteilung einer Variante in der Stichprobe dargestellt, die eine Erhöhung des Risikos der MS-Erkrankung bei Vorliegen mindestens einer Risikovariante um das 2,67-Fache zeigt (Odds Ratio [OR] =2,67). Dies entspricht etwa der OR des HLA-DRB1*1501Risikoallels. Die Frequenz des Risikoallels in der Patientenstichprobe beträgt hierbei $40 \%$, in der Kontrollstichprobe $20 \%$ (entsprechend der Frequenz des HLA-Risikoallels in der europäischen Bevölkerung). Bei dieser Effektstärke und Allelfrequenz in der Stichprobe sind 25 Patienten und 25 Kontrollprobanden notwendig, um einen nominal-signifikanten $p$-Wert zu erlangen $(p<0,05)$. $\bullet \mathbf{A b b}$. $\mathbf{1 b}$ zeigt die Verteilung einer Variante bei einer beobachteten OR von 1,12 in der Stichprobe. Dies entspricht etwa der mittleren beobachteten Effektstärke aller bisher etablierten MS-Risikovarianten. Die Allelfrequenz in der Patientenstichprobe beträgt hier 22\%, in der Kontrollstichprobe $20 \%$. Um hier ein nominal-signifikantes Ergebnis zu erreichen, sind 1600 Patienten und 1600 Kontrollen nötig. (Die Darstellung wurde zur Vereinfachung auf 400 Patienten und 400 Kontrollen limitiert.) Um genomweite $\mathrm{Si}$ gnifikanz $(p<5 \times 10-8)$ zu erreichen, sind im Fall des Szenarios A (OR 2,67) etwa 160 Patienten und 160 Kontrollen notwendig, im Fall von Szenario B $(O R$ 1,12) jedoch etwa 12.500 Patienten und 12.500 Kontrollprobanden

hang zwischen der Frequenz einer genetischen Variante, z. B. eines Einzelbasenaustausches (,single nuleotide polymorphism [SNP]“), und bestimmten Phänotypen. Der Phänotyp kann ein qualitatives Merkmal sein, etwa das Vorkommen einer Erkrankung wie MS. Dabei wird die
Frequenz von genetischen Varianten in Patienten mit der in nichterkrankten Kontrollprobanden verglichen ( $\bullet$ Abb. 1). Die resultierenden Effekte von genetischen Varianten auf das Krankheitsrisiko werden gewöhnlich als „Odds Ratios $[\mathrm{OR}]$ “ angegeben, die bei Erkrankungen mit geringer Prävalenz wie der MS dem „relativen Risiko" entsprechen. Zum Beispiel erhöht eine genetische Variante mit einer OR von 2,0 oder 1,3 das Risiko der MS-Erkrankung um das 2-fache bzw. das 1,3-fache. Bei genetischen Assoziationsstudien in Familien wird getestet, ob bestimmte Varianten häufiger an erkrankte Kinder weitergegeben werden. Diese Methode hat jedoch eine geringere Trennschärfe („power") als Fall-Kontroll-Studien und wird deshalb bei Assoziationsstudien zunehmend weniger eingesetzt. Neben qualitativen Merkmalen kann der $\mathrm{Zu}$ sammenhang von genetischen Markern mit quantitativen Merkmalen untersuchen werden, z. B. mit dem Erkrankungsalter oder dem Behinderungsgrad in der Patientenstichprobe.

Typischerweise gibt es in genetischen Assoziationsstudien zwei Ansätze: Zum einen können in sog. Kandidatengenstudien gezielt genetische Varianten in Genen oder einer Gruppe von Genen getestet werden, die für eine Erkrankung funktionell interessant erscheinen, d. h. die z. B. in In-vitro- oder In-vivo-Studien pathophysiologisch mit der Erkrankung in Verbindung gebracht worden sind. Seit 2005 gibt es neben dem KandidatengenAnsatz die Möglichkeit, in genomweiten Assoziationsstudien (GWAS) „hypothesenfrei" hunderttausende bis mehrere Millionen SNPs, die über das gesamte Genom verteilt sind, in einem einzigen Experiment zu testen. Hierbei werden genetische Varianten, die mit einer bestimmten Mindestfrequenz (häufig $1 \%$ oder $5 \%$, sog. Polymorphismen) in der Referenzpopulation vorkommen, getestet, d. h. wenig häufige $(<1 \%)$ genetische Varianten werden normalerweise nicht erfasst. Der bioinformatisch und statistisch sehr anspruchsvolle GWAS-Ansatz erfordert eine stringente Korrektur für multiples Testen. Gewöhnlich wird, unter der Annahme von bis zu 1 Mio. unabhängiger Genorte im menschlichen Genom, ein Signifikanzniveau von $5 \times 10^{-8}$ angenommen. Häufig werden in diesen Studien statistisch „interessante“ Assoziationssignale anschließend in weiteren Stichproben derselben oder anderer Populationen gezielt validiert. Während der Kandidatengen-Ansatz in vielen komplex-genetischen Erkrankungen zu der Identifizie- 
Hier steht eine Anzeige.

黛 Springer 


\section{Infobox 1 Internetadressen \\ - www.msgene.org \\ www.msgene.org/largescale.asp \\ www.pdgene.org}

rung einiger weniger Risikogene geführt hat, die häufig die größten Effekte in den jeweiligen Erkrankungen zeigen, hat der genomweite Assoziationsansatz in den letzten Jahren zu der Identifizierung vieler weiterer Risikogene geführt, die typischerweise jedoch nur kleine bis moderate Effekte haben. Diese Situation trifft auch auf die MS zu und soll im Folgenden dargestellt werden.

\section{HLA}

Vor fast 40 Jahren wurde in der Anfangsphase der Kandidatengenforschung eine Assoziation von MS mit der HLA („human leucocyte antigen“ oder Transplantationsantigen)-Genregion auf Chromosom 6 beschrieben $[17,21]$, die sich in praktisch allen Nachfolgestudien in anderen Populationen bestätigt hat und die bis heute den stärksten Effekt auf das Erkrankungsrisiko aufweist. Die sehr genreiche HLA-Region (>160 proteinkodierende Gene) ist mit über 44.000 genetischen Varianten die variabelste Region des Genoms. Zugleich sind sehr viele genetische Varianten, die oft sogar in verschiedenen Genen liegen, miteinander gekoppelt, d. h. sie werden häufig miteinander vererbt. Etwa 40\% der Gene der HLARegion kodieren für Proteine, die im Immunsystem eine Rolle spielen. Deshalb ist es sehr schwierig, die tatsächlich funktionell relevanten Varianten oder Kombinationen von Varianten, sog. Haplotypen, zu identifizieren, die der Assoziation mit MS zugrunde liegen. Inzwischen hat sich herausgestellt, dass in den meisten Populationen europäischen Ursprungs sowie in der afroamerikanischen Population das sog. HLA-DRB1*1501-Allel, das einen Haplotyp darstellt, als Hauptrisikofaktor wirkt (zur Übersicht s. [28]). Es erhöht das Risiko einer MS-Erkrankung um das 2- bis 3-fache (OR ca. 2,6) und erklärt etwa $10 \%$ der Erblichkeit [26]. HLA-DRB1 gehört zu den HLA-Klasse-II-Molekülen, die verantwortlich sind für die Präsentation von extrazellulären, potenziell patho- genen Peptiden auf antigenpräsentierenden Zellen und damit für die Aktivierung von $\mathrm{CD} 4{ }^{+} \mathrm{T}-$ Zellen. Strukturelle Studien haben postuliert, dass der Haplotyp von $H L A-D R B 1^{\star} 1501$ durch eine Veränderung der Form und Ladung bestimmter Strukturen an der Peptidbindungsstelle des HLA-II-Moleküls die Antigenbindung und -präsentation verändert [29].

Aufgrund der vielen immunologisch relevanten Gene in der HLA-Region wurde in der Folge nach zusätzlichen, von $H L A-D R B 1^{\star} 1501$ unabhängigen Risikovarianten für MS gesucht. In den letzten Jahren konnten neben $H L A-D R B 1^{\star} 1501$ weitere Assoziationen mit HLA-Klasse-I-Genen nachgewiesen werden (z. B. [7]). HLA-I-Komplexe sind verantwortlich für die Präsentation von intrazellulären Molekülen an der Oberfläche der jeweiligen Zellen und aktivieren zytotoxische $\mathrm{CD}^{+}{ }^{+} \mathrm{T}-$ Zellen. Eine Rolle von $\mathrm{CD}^{+}{ }^{+}$Zellen für die Pathogenese der MS wird auch durch funktionelle Daten erhärtet (z. B. [10]). Der genaue Wirkmechanismus der MS-assoziierten genetischen Haplotypen in HLA-I- und HLAII-Molekülen in dem pathogenetischen Prozess ist bisher jedoch kaum verstanden.

\section{Andere Kandidatengenstudien}

Nach der frühen und erfolgreichen Identifizierung der HLA-Genregion als Risikofaktor für MS wurden über mehr als 30 Jahre in hunderten von Studien mehr als 600 andere, zumeist immunologisch interessante Gene auf ihre Assoziation mit MS getestet und dutzende potenzielle Risikogene postuliert (für einen Überblick über die Datenlage s. www.msgene.org, - Infobox 1). Zu den am häufigsten untersuchten Genen gehören $A P O E$, CCR5, CTLA4, IFNG, ICAM1, IGH@, IL1A und B, IL4, IL6 und IL10, ILIRN, MBP,TRB@ sowie mitochondriale Gene. Für keines dieser Gene gibt es jedoch bisher überzeugende Evidenzen für eine Assoziation mit MS. Gründe für die fehlende Identifizierung von weiteren Risikogenen mithilfe des Kandidatengen-Ansatzes sind vielfältig: So wurden etwa Effektgrößen weiterer potenzieller genetischer Risikovarianten überschätzt und deshalb zu kleine Stichproben untersucht. Begrenzte
Einblicke in die pathogenetischen Mechanismen führten zur Wahl von ungeeigneten Kandidatengenen, und das genaue Spektrum genetischer Variationen im Genom war bis zur Vollendung verschiedener Genomprojekte nur unzureichend bekannt. Liberale Signifikanzniveaus ohne Korrektur für multiples Testen führten häufig zu initial falsch-positiven Ergebnissen, und es kam zu falsch-positiven und -negativen Ergebnissen durch unkontrollierte Verzerrungen („Bias“), etwa durch eine ethnische Durchmischung der Stichproben („Populationsstratifikation“). Eine bemerkenswerte Ausnahme aus dem Ende der Kandidatengen-Ära stellt die Identifizierung des MS-Risikogens $I L 7 R$ dar, das für die $\alpha$-Kette des Interleukin-7-Rezeptors kodiert [11, 19, 30].

\section{Genomweite Assoziationsstudien}

Im Jahr 2007 wurde die erste GWAS in MS veröffentlicht, die die Assoziation mit dem HLA-Locus und IL7R bestätigen konnte und zugleich eine Assoziation mit IL2RA beschrieb ([12], • Tab. 1). Seitdem sind 8 GWAS publiziert worden, die rund 50 MS-Risiko-Loci berichteten, die genomweit signifikante $\left(\mathrm{p}<5 \times 10^{-8}\right)$ Evidenz für eine Assoziation zeigten (für eine Übersicht s. $\bullet$ Tab. 1). Dabei hat sich mit immer größer werdender Studiengröße und damit größerer Power auch die Anzahl der beschriebenen und im Folgenden validierten MS-Risikogene vergrößert. Des Weiteren wurden in anderen umfassenden Assoziationstudien, z. B. großangelegten GWAS-Validierungsstudien oder GWAS-Metaanalysen, weitere MS-Risikogene gefunden (für eine Übersicht s. www.msgene.org/largescale.asp, - Infobox 1; [31]).

Die kürzlich veröffentlichte größte GWAS [26], die Proben verschiedener europäischer Länder sowie der USA einschloss, hat in ca. 10.000 Fällen und ca. 17.000 Kontrollen die Assoziation mit 23 der zuvor postulierten MS-Genen bestätigt und außerdem 29 neue potenzielle MS-Gene beschrieben (• Tab. 1). Letztere wurden in der Studie bereits in den Datensätzen früherer GWAS validiert. Dennoch bedarf es weiterer Daten in unabhängigen Stichproben, um die Rol- 
le dieser neuen Genorte für das MS-Risiko zweifelsfrei zu klären und z. B. die funktionell relevanten Sequenzvarianten ausfindig zu machen. Die GWAS konnte ebenfalls die von HLA-DRB1 unabhängige Assoziation von HLA-Klasse-I-Genen bestätigen. Insgesamt haben die durch die GWAS der letzten Jahre identifizierten Genorte kleine bis moderate Effekte auf das MS-Risiko. Die mittlere Effektstärke (OR) beträgt ca. 1,12 (1,1-1,3), d. h. sie beträgt nur ca. ein Zehntel des HLA-Effekts. Das ist vergleichbar mit der Effektstärke von GWAS-Loci anderer komplexgenetischer Erkrankungen [14]. GALC, CD86 und PTGER4 haben mit ORs von 1,26, 1,21 und 1,21 die größten Effekte unter den durch GWAS identifizierten MS-Risiko-Loci. Bei Kombination aller bis heute identifizierter Risiko-Loci einschließlich des HLA-Locus sind etwa 17\% der Erblichkeit erklärbar [26].

Trotz individuell kleiner Effektstärken bieten die identifizierten Genorte einen einzigartigen Einblick in die genetische Architektur der Erkrankung und mögliche zugrunde liegende Pathomechanismen. So sind die überwiegende Mehrzahl der MS-Risikogene, die in der letzten großen GWAS gefunden wurden [26], beteiligt an immunologischen Prozessen, besonders zytokinabhängigen Signalwegen und anderen Signaltransduktionswegen von immunologischer Relevanz. Zwei weitere Gene (CYP27B1, CYP24A1), die eine Rolle im Vitamin-D-Stoffwechsel spielen, zeigen ebenfalls eine Assoziation, was aufgrund des lange postulierten Umwelteffekts von Vitamin D und Sonneneinstrahlung auf das MS-Risiko (zur Übersicht s. [23]) sehr interessant ist. Lediglich zwei Gene, die eine Assoziation mit MS zeigen (GALC, KIF21B), könnten in primär neurodegenerativen Prozessen eine Rolle spielen. Somit sind die genetischen Evidenzen für einen primär neurodegenerativen Pathomechanismus bei der MS bisher eher schwach [26].

Die genaue Aufklärung der zugrunde liegenden Funktion der identifizierten MS-Risiko-Gene, z. B. durch In-vitro- oder In-vivo-Experimente oder durch Untersuchung von Endophänotypen der MS, kann unser Verständnis von der Pathophysiologie der MS vertiefen und ggf. erweitern. Dies kann jedoch unter Um-

Nervenarzt 2012 $\cdot 83: 705-713 \quad$ DOI 10.1007/s00115-011-3438-9

C) Springer-Verlag 2012

\section{C.M. Lill · F. Zipp}

\section{Genetisches Profil der Multiplen Sklerose: Risikogene und die "dunkle Materie"}

\section{Zusammenfassung}

Multiple Sklerose (MS) ist eine multifaktorielle Erkrankung, die auf der Grundlage von Wechselwirkungen genetischer und umweltbedingter Faktoren entsteht. Sie tritt gehäuft familiär auf, die Vererbung folgt aber nicht den klassischen mendelschen Regeln. Das Risiko einer MS-Erkrankung wird genetisch vielmehr beeinflusst durch dutzende bis hunderte von genetischen Varianten, die auch in der Normalbevölkerung vorkommen. Den größten, seit 40 Jahren bekannten Risikoeffekt haben genetische Varianten in der HLA-Region, die das Risiko einer MS um das Zwei- bis Dreifache erhöhen. In den letzten Jahren konnten durch genomweite Assoziationsstudien (GWAS) fast 60 weitere Risikofaktoren identifiziert werden. Jeder dieser genetischen Fak- toren führt zu einer moderaten Erhöhung des Risikos (mit Odds Ratios von 1,1-1,3). Die zurzeit bekannten genetischen Risikofaktoren machen jedoch nur einen geringen Anteil der Erblichkeit der MS aus. Die durch den GWASAnsatz nicht identifizierbaren genetischen Risikofaktoren, gelegentlich auch als „dunkle Materie" bezeichnet, sind möglicherweise durch andere vererbbare Faktoren bedingt wie etwa strukturelle Variationen im Genom, seltene Sequenzvarianten sowie vererbte epigenetische Modifikationen.

Schlüsselwörter

Multiple Sklerose · Genetik - Genomweite Assoziationsstudien · Familiäre Häufung · HLA-Region

\section{The genetic profile of multiple sclerosis: risk genes and the "dark matter"}

\section{Summary}

Multiple sclerosis (MS) is a genetically complex disease caused by the interplay of genetic and environmental factors. While it shows substantial familial accumulation, there is no evidence for typical Mendelian transmission within families. Instead, MS risk is likely governed by dozens to hundreds of genetic variants, which can also be present in the general population. The strongest genetic risk factor for MS was identified 40 years ago and lies within the HLA locus. It increases the risk of developing MS by two- to threefold. Within the last few years, genome-wide association studies (GWAS) have led to the identification of nearly 60 additional genetic risk loci. Each of these loci yields a modest to moderate risk increase (odds ratios of 1.1-1.3). Even in combination, however, the currently known risk variants merely account for a small fraction of the disease's heritability. It is likely that a major fraction of genetic MS risk that cannot be explained by GWAS, sometimes termed the "dark matter" of GWAS, is caused by other factors, such as structural variations of the genome, rare sequence variants, or inherited epigenetic modifications.

\section{Keywords}

Multiple sclerosis - Genetics - Genome-wide association study · Familial aggregation · HLA locus ständen ein schwieriger Prozess sein. So ist es selbst bei anderen, eindeutig monogenen Erkrankungen oder auch bei der seit Jahrzehnten bekannten HLA-Assoziation der MS immer noch nicht gelungen, eine eindeutige Funktionsbeziehung aufzuzeigen.

\section{Gemeinsame Risikofaktoren autoimmunologischer Erkrankungen}

In diesem Zusammenhang ist es interessant, dass einige der MS-Risiko-Loci auch in anderen Autoimmunerkrankungen Assoziationen zeigen wie etwa in Typ-1-Diabetes, systemischem Lupus erythematodes, rheumatoider Arthritis, Psoriasis und chronisch entzündlichen Darmerkrankungen [14]. Keine Überlappung besteht hingegen zwischen MS und den bisher 


\begin{tabular}{|c|c|c|c|c|}
\hline GWAS & $\begin{array}{l}\text { Population(en) GWAS } \\
\text { (Validierungsphase) }\end{array}$ & $\begin{array}{l}\text { Studiendesign und Stich- } \\
\text { probengröße GWAS (Vali- } \\
\text { dierungsphase) }^{b}\end{array}$ & $\begin{array}{l}\text { Anzahl SNPs } \\
\text { GWAS (Validie- } \\
\text { rungsphase) }\end{array}$ & Postulierte genetische Locic \\
\hline IMSGC [12] & $\begin{array}{l}\text { USA, Großbritannien } \\
\text { (USA, Großbritannien) }\end{array}$ & $\begin{array}{l}\text { FB: } 931 \text { trios, CC: } 931 / 2431 \\
\text { (FB: } 609 \text { trios, CC: } 2322 / 2987 \text { ) }\end{array}$ & $334.923(110)$ & HLA, IL2RA, IL7RA \\
\hline $\begin{array}{l}\text { Comabella } \\
\text { et al. [5] }\end{array}$ & $\begin{array}{l}\text { Spanien } \\
\text { (Spanien, USA) }\end{array}$ & $\begin{array}{l}\text { CC: } 242 / 242 \\
\text { (CC: } 553 / 1033)\end{array}$ & $428.867(320)$ & HLA, $13 q 31.3$ \\
\hline $\begin{array}{l}\text { Aulchenko } \\
\text { et al. [1] }\end{array}$ & $\begin{array}{l}\text { Niederlande } \\
\text { (Niederlande, Schweden, } \\
\text { Kanada) }\end{array}$ & $\begin{array}{l}\text { CC: } 45 / 195 \\
\text { (FB: } 1318 \text { trios, CC: } \\
1316 / 1423 \text { ) }\end{array}$ & $211.555(1)$ & KIF1B \\
\hline $\begin{array}{l}\text { Baranzini } \\
\text { et al. [3] }\end{array}$ & $\begin{array}{l}\text { USA, Schweiz, Nieder- } \\
\text { lande (-) }\end{array}$ & CC: 978/883 (-) & $551.642(-)$ & HLA, GPC5 \\
\hline $\begin{array}{l}\text { ANZgene } \\
{[2]}\end{array}$ & $\begin{array}{l}\text { Australien, Neuseeland, } \\
\text { USA, Großbritannien } \\
\text { (Australien, Neuseeland) }\end{array}$ & $\begin{array}{l}\text { CC: } 1618 / 3413 \\
\text { (CC: } 2256 / 2310)\end{array}$ & $302.098(91)$ & $\begin{array}{l}\text { HLA, CD40, CYP27B1, CD58, EVI5, IL2RA, CLEC16A, IL7R, } \\
\text { TYK2 }\end{array}$ \\
\hline $\begin{array}{l}\text { Jakkula et } \\
\text { al. [16] }\end{array}$ & $\begin{array}{l}\text { Finnland } \\
\text { (Finnland, Norwegen, Dä- } \\
\text { nemark sowie publizierte } \\
\text { GWAS-Datensätze) }\end{array}$ & $\begin{array}{l}\text { CC: 68/136 } \\
\text { (CC: } 4570 / 10139)\end{array}$ & $297.343(28)$ & HLA, STAT3 \\
\hline $\begin{array}{l}\text { Nischwitz, } \\
\text { et al. [22] }\end{array}$ & Deutschland (-) & CC: 592/825 (-) & $\sim 300.000^{\mathrm{d}}(-)$ & HLA, VAV2, ZNF433 \\
\hline $\begin{array}{l}\text { Sanna et al. } \\
\text { [24] }\end{array}$ & $\begin{array}{l}\text { Sardinien } \\
\text { (Sardinien) }\end{array}$ & $\begin{array}{l}\text { CC: } 882 / 872 \\
\text { (CC: } 1775 / 2005)\end{array}$ & $6.607 .266(9)$ & HLA, CBLB \\
\hline IMSGC [26] & $\begin{array}{l}\text { Europe und USA } \\
\text { (publizierte GWAS-Daten- } \\
\text { sätze) }\end{array}$ & $\begin{array}{l}\text { CC: } 9772 / 17376 \\
\text { (CC: } 4355 / 7490)\end{array}$ & $465.434(102)$ & $\begin{array}{l}\text { HLA, AGAP2, BACH2, BATF, C1 orf106, C3orf1, CD5, CD58, } \\
\text { CD86, CLEC16A, CLECL1, CYP24A1, DKKL1, EVI5, GALC, } \\
\text { HHEX, IL12B, IL2RA, IL7R, IL22RA2, IRF8, MALT1, MAPK1, } \\
\text { MERTK, MMEL1, MPV17L2, MYB, MYC, NCOA5, NFKBIZ, } \\
\text { PLEK, PTGER4, RGS1, SCO2, SLC30A7, SP140, STAT3, } \\
\text { TAGAP, THEMIS, TNFRSF1A, TNFSF14, ZFP36L1, ZMIZ1, } \\
\text { ZNF767, 2p21, 3p24.1 }\end{array}$ \\
\hline \multicolumn{5}{|c|}{ 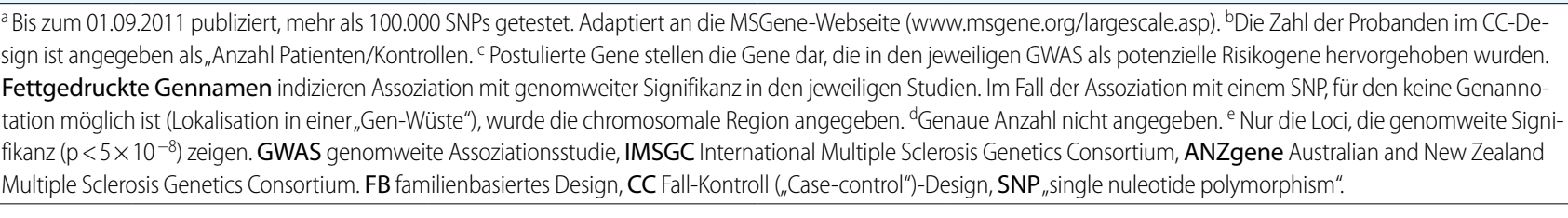 } \\
\hline
\end{tabular}

bekannten Genorten in neurodegenerativen Erkrankungen wie Alzheimer, frontotemporaler Demenz oder amyotropher Lateralsklerose [18]. Lediglich in der Parkinson-Krankheit wurde vor kurzem eine Assoziation mit dem HLA-Locus (HLA$D R A, H L A-D R B 5$ ) berichtet, die jedoch eine wesentlich kleinere Effektstärke aufweist (OR ca. 1,3; für weitere Details s. www.pdgene.org, $\bullet$ Infobox 1 ).

\section{Die „dunkle Materie“}

Es kann erwartet werden, dass durch noch größere Studien und Studien in anderen Populationen weitere MS-Risikogene identifiziert werden. Die meisten Loci mit moderaten Effekten sind durch die vorausgegangenen GWAS vermutlich bereits identifiziert wurden, sodass durch größere Stichproben v. a. neue Loci mit noch kleineren Effektstärken (ORs < 1,15) identifiziert werden. Auch die Testung weiterer Marker wird höchstwahrscheinlich zur Identifizierung zusätzlicher Risikogene führen. In der kürzlich veröffentlichten GWAS [26] wurden „nur“ ca. 500.000 SNPs untersucht. Es ist seit einiger Zeit möglich, aufgrund von Informationen aus Referenzpopulationen über den Grad der Kopplung der untersuchten SNPs bis zu 10 Mio. weitere nicht direkt getestete SNPs in den Probanden zu berechnen, d. h. zu ,imputieren“, und diese in der Stichprobe auf Assoziation mit der Erkrankung zu testen. Es kann erwartet werden, dass die Imputation aller verfügbaren GWAS-Datensätze inklusive der letzen großen GWAS sowie die Zusammenführung dieser Datensätze mittels Metaanalyse die Anzahl der MS-Risiko-Loci noch einmal substanziell erhö- hen wird, was auch zur Identifikation von Genen mit moderaten Effektstärken (mit ORs bis zu ca. 2) führen könnte. Des Weiteren haben die meisten bisherigen Kandidatengenstudien sowie alle GWAS bisher ausschließlich Populationen europäischen Ursprungs getestet. GWAS in anderen Ethnizitäten, etwa in asiatischen oder arabischen Populationen, könnten wertvolle Einblicke in die potenzielle ätiologische Heterogenität der MS gewähren.

Die Schätzung, dass bisher nur etwa 17\% der Erblichkeit der MS durch die identifizierten Risiko-Loci bekannt ist, entspricht der Situation in anderen komplex-genetischen Erkrankungen. Berechnungen haben ergeben, dass im besten Fall nur bis zu 50\% der Gesamterblichkeit durch mithilfe von GWAS identifizierten Risikogenorte erklärt werden kann - mit anderen Worten, der größte Anteil der 
Hier steht eine Anzeige.

黛 Springer 
Genetik an der Krankheitsvarianz bleibt unerklärt. Dieses Phänomen wurde als „the dark matter of GWAS“ („dunkle Materie der GWAS“) bezeichnet. Das bedeutet, dass zusätzliche genetische oder erbliche Risikofaktoren existieren müssen, aber GWAS nicht geeignet sind, sie zu detektieren $[4,20]$. Eine Reihe von Vermutungen sind aufgestellt worden, woraus diese „dunkle Materie“ bestehen könnte (zur Übersicht s. z. B. [9, 20]):

- Es könnten strukturelle genetische Varianten (Deletionen, Duplikationen und Inversionen) eine Rolle spielen.

- Es könnte genetische Varianten geben, die große Effektstärken haben, aber mit einer Frequenz in der Bevölkerung von $<<1 \%$ selten vorkommen. Beispiele dafür gibt es in anderen Erkrankungen, z. B. GBA-Gen in der Parkinson-Erkrankung (s. www. pdgene.org; [31]). Diese beiden Möglichkeiten werden in Kürze mit dem Einsatz neuer Hochdurchsatz-Sequenziertechnologien, dem sog. „next generation sequencing", überprüfbar sein. Dabei können alle Exone eines Genoms („whole-exome sequencing“) bzw. das ganze Genom (,whole-genome sequencing") komplett durchsequenziert werden und nach krankheitsauslösenden und prädisponierenden Sequenzvarianten abgesucht werden. Dies ermöglicht neben der Erfassung bereits bekannter Polymorphismen eine Identifizierung und Testung bis dahin unbekannter struktureller oder seltener Varianten.

- An der „dunklen Materie“ könnten darüber hinaus epigenetische Effekte, die von der Elterngeneration weitergegeben werden und sequenzunabhängig sind, eine Rolle spielen, z. B. durch DNA-Methylierung und Histonmodifikationen. Auch dies ist in zunehmendem Maße durch „next generation sequencing" erfassbar.

- Gen-Gen- und Gen-Umwelt-Interaktionen könnten eine Rolle spielen, die Effekte zeigen, die mit den bisher eingesetzten, vergleichsweise einfachen statistischen Assoziationsmodellen nicht zu erfassen sind. Gen-UmweltInteraktionen werden am schwierigsten zu untersuchen sein durch die ungenügende Kenntnis und Erhe- bung von relevanten Umweltfaktoren, schwer zu kontrollierenden Bias (etwa durch inadäquates Studiendesign, fehlerhafte Datenerhebung, Störvariablen) sowie geringe Power in den Analysen.

\section{Fazit für die Praxis}

Würden wir die genetische Architektur der MS vollständig verstehen, wäre prinzipiell die DNA-Sequenz der optimale Biomarker, da sie unverändert seit Lebensbeginn vorhanden und erfassbar ist. Aufgrund der dort enthaltenen Information könnte eine individuelle Prädiktion des MS-Risikos erstellt werden, und es könnten abgestimmte Präventionsmaßnahmen ergriffen werden. Damit wäre das oft gebrauchte Schlagwort der „personalisierten Medizin“ in der Genetik Realität geworden. Wie oben ausgeführt, kennen wir jedoch bisher nur einen kleinen Anteil des genetischen Profils der Erkrankung. Aus diesem Grund ist es zurzeit nicht möglich, individuelle Vorhersagen für das MS-Risiko abzuleiten. Ein erster Versuch solch einer Vorhersage mit 16 zu der Zeit bekannten Risikomarkern konnte Probanden in Risikokategorien einteilen, von denen die Kategorie mit dem höchsten genetischen Risikoprofil ein 5-fach erhöhtes Risiko einer MS hatte [15], was jedoch bei dem eher geringen Lebenszeitrisiko von MS eine nur geringe Bedeutung hat: Ausgehend von einer Prävalenz von 0,1-0,3\% ergäbe das im Falle der höchsten Risikokategorie lediglich eine Erhöhung des Lebenszeitrisikos auf 0,5-1,5\%. Im Vergleich hierzu wird das Risiko von Verwandten 1. Grades bei MS-Patienten auf 3-5\% geschätzt (s. oben). Es kann erwartet werden, dass mit der Erweiterung unserer Kenntnis über die genetische Architektur der MS bessere Vorhersagen über das individuelle Risiko möglich sind. Sollten tatsächlich verschiedene seltene Varianten mit höheren Effektstärken eine Rolle für das MS-Risiko spielen, würde das die Möglichkeiten einer Prädiktion entscheidend verbessern [25]. Zum jetzigen Zeitpunkt jedoch erbringt eine Vorhersage des Erkrankungsrisikos durch genetisches Testen im Falle der MS keinen Informations- gewinn. Die Nutzung von „Direct-to-consumer"-Angeboten einer genetischen Testung mit dem Ziel der Prädiktion der MS-Erkrankungswahrscheinlichkeit mag auf Seiten der Beteiligten zu falschen Schlussfolgerungen führen und sollte ärztlicherseits nicht unterstützt werden. Doch auch wenn die mit einem erhöhten MS-Risiko assoziierten genetischen Varianten jeweils nur kleine Effekte haben, so geben sie dennoch entscheidende Hinweise darauf, welche Gene funktionell bei der Entstehung der MS involviert sind. Deshalb könnten die gewonnenen Erkenntnisse und die verfügbare Methodik schon jetzt hilfreich sein, verbesserte und neue Therapiemöglichkeiten zu entwickeln. So ist bemerkenswert, dass einige der identifizierten MS-Risiko-Gene bereits Angriffspunkte für verfügbare MSTherapien darstellen, d. h. VCAM1 in der Natalizumab-Therapie, IL2RA in der Daclizumab-Therapie und einige Gene, die in Interferon- $\beta$-beeinflussten Signalwegen eine Rolle spielen. Die neuen MS-Risiko-Gene, die wir mit einem internationalen Netzwerk identifizieren konnten [26], unterstreichen in jedem Fall die wichtige Rolle des Immunsystems in der Multiplen Sklerose.

\section{Korrespondenzadresse}

\section{Prof. Dr. F. Zipp}

Klinik und Poliklinik für Neurologie,

Johannes-Gutenberg-Universität

Langenbeckstr. 1, 55131 Mainz

zipp@uni-mainz.de

Danksagung. Unser Dank gilt allen Mitarbeitern und Kollaborateuren, die zur Erstellung und Aktualisierung der MSGene-Datenbank beigetragen haben, besonders Dr. Lars Bertram, Dr. Esther Meissner, Maria Liebsch und Ute Zauft. Wir danken Dr. Lars Bertram für hilfreiche Diskussionen und das kritische Lesen des Manuskripts. Die MSGene-Datenbank wird gefördert durch EMD Serono, Inc.

Interessenskonflikt. Der korrespondierende Autor gibt an, dass kein Interessenkonflikt besteht.

\section{Literatur}

1. Aulchenko YS, Hoppenbrouwers IA, Ramagopalan SV et al (2008) Genetic variation in the KIF1B locus influences susceptibility to multiple sclerosis. Nat Genet 40:1402-1403 
2. Australia and New Zealand Multiple Sclerosis Genetics Consortium (ANZgene) (2009) Genomewide association study identifies new multiple sclerosis susceptibility loci on chromosomes 12 and 20. Nat Genet 41:824-828

3. Baranzini SE, Wang J, Gibson RA et al (2009) Genome-wide association analysis of susceptibility and clinical phenotype in multiple sclerosis. Hum Mol Genet 18:767-778

4. Bertram L, Lill CM, Tanzi RE (2010) The genetics of Alzheimer disease: back to the future. Neuron 68:270-281

5. Comabella M, Craig DW, Camiña-Tato M et al (2008) Identification of a novel risk locus for multiple sclerosis at $13 q 31.3$ by a pooled genomewide scan of 500,000 single nucleotide polymorphisms. PloS One 3:e3490

6. Compston A, Coles A (2008) Multiple sclerosis. Lancet 372:1502-1517

7. Cree BAC, Rioux JD, McCauley JL et al (2010) A major histocompatibility Class I locus contributes to multiple sclerosis susceptibility independently from HLA-DRB1*15:01. PloS One 5:e11296

8. Dyment DA, Cader MZ, Willer CJ et al (2002) A multigenerational family with multiple sclerosis. Brain 125:1474-1482

9. Eichler EE, Flint J, Gibson G et al (2010) Missing heritability and strategies for finding the underlying causes of complex disease. Nat Rev Genet 11:446-450

10. Friese MA, Jakobsen KB, Friis L et al (2008) Opposing effects of $\mathrm{HLA}$ class I molecules in tuning autoreactive $\mathrm{CD} 8{ }^{+} \mathrm{T}$ cells in multiple sclerosis. Nat Med 14:1227-1235

11. Gregory SG, Schmidt S, Seth P et al (2007) Interleukin 7 receptor alpha chain (IL7R) shows allelic and functional association with multiple sclerosis. Nat Genet 39:1083-1091

12. Hafler DA, Compston A, Sawcer S et al (2007) Risk alleles for multiple sclerosis identified by a genomewide study. N Engl J Med 357:851-862

13. Haghighi S, Andersen O, Nilsson S et al (2006) A linkage study in two families with multiple sclerosis and healthy members with oligoclonal CSF immunopathy. Mult Scler 12:723-730

14. Hindorff LA, Sethupathy $P$, Junkins HA et al (2009) Potential etiologic and functional implications of genome-wide association loci for human diseases and traits. Proc Nat Acad Sci U S A 106:9362-9367

15. De Jager PL, Chibnik LB, Cui J et al (2009) Integration of genetic risk factors into a clinical algorithm for multiple sclerosis susceptibility: a weighted genetic risk score. Lancet Neurol 8:1111-1119

16. Jakkula E, Leppä V, Sulonen AM et al (2010) Genome-wide association study in a high-risk isolate for multiple sclerosis reveals associated variants in STAT3 gene. Am J Hum Genet 86:285-291

17. Jersild C, Svejgaard A, Fog T (1972) HL-A antigens and multiple sclerosis. Lancet 1:1240-1241

18. Lill CM, Bertram L (2012) Towards unveiling the genetics of neurodegenerative diseases. Sem Neurol (in press)

19. Lundmark F, Duvefelt K, lacobaeus E et al (2007) Variation in interleukin 7 receptor alpha chain (IL7R) influences risk of multiple sclerosis. Nat Genet 39:1108-1113

20. Manolio TA, Collins FS, Cox NJ et al (2009) Finding the missing heritability of complex diseases. Nature 461:747-753

21. Naito S, Namerow N, Mickey MR, Terasaki PI (1972) Multiple sclerosis: association with $\mathrm{HL}-\mathrm{A} 3$. Tissue Antigens 2:1-4
22. Nischwitz S, Cepok S, Kroner A et al (2010) Evidence for VAV2 and ZNF433 as susceptibility genes for multiple sclerosis. J Neuroimmunol 227:162-166

23. Pierrot-Deseilligny C, Souberbielle JC (2010) Is hypovitaminosis $D$ one of the environmental risk factors for multiple sclerosis? Brain 133:1869-1888

24. Sanna S, Pitzalis M, Zoledziewska M et al (2010) Variants within the immunoregulatory CBLB gene are associated with multiple sclerosis. Nat Genet 42:495-497

25. Sawcer S, Ban M, Wason J, Dudbridge F (2010) What role for genetics in the prediction of multiple sclerosis? Ann Neurol 67:3-10

26. Sawcer S, Hellenthal G, Pirinen M et al (2011) Genetic risk and a primary role for cell-mediated immune mechanisms in multiple sclerosis. Nature 476:214-219

27. Sawcer $S$ (2008) The complex genetics of multiple sclerosis: pitfalls and prospects. Brain 131:3118 3131

28. Schmidt $H$, Williamson D, Ashley-Koch A (2007) HLA-DR15 haplotype and multiple sclerosis: a HuGE review. Am J Epidemiol 165:1097-1109

29. Smith KJ, Pyrdol J, Gauthier L et al (1998) Crystal structure of HLA-DR2 (DRA*0101, DRB1*1501) complexed with a peptide from human myelin basic protein. J Exp Med 188:1511-1520

30. Zhang Z, Duvefelt K, Svensson F et al (2005) Two genes encoding immune-regulatory molecules (LAG3 and IL7R) confer susceptibility to multiple sclerosis. Genes Immun 6:145-152

31. Lill CM, Roehr JT, McQueen M et al (2012) Comprehensive research synopsis and systematic meta-analyses in Parkinson's Disease genetics: The PDGene database (Manuskript unter Begutachtung)

\section{Neuer Mechanismus des Vergessens}

Berner Forscher haben im Gehirn einen Mechanismus nachgewiesen, der für das Vergessen von Informationen verantwortlich sein könnte. Dabei spielen die Astrozyten und ein vom Gehirn selbst produzierter cannabisähnlicher Stoff eine wichtige Rolle. Aktiviert eine vorgeschaltete Nervenzelle eine Synapse, nachdem die nachgeschaltete Nervenzelle schon einen elektrischen Impuls generiert hat, kommt diese Information zu spät. Wiederholt sich dieser Vorgang häufig, sendet die nachgeschaltete Nervenzelle dämpfende Botenstoffe aus. Dies bewirkt eine Schwächung der Verbindung zwischen den beteiligten Nervenzellen. Die Forscher haben festgestellt, dass dieses Signal über die Astrozyten übermittelt wird. Astrozyten umschließen die Kontaktstelle der beiden Nervenzellen. Sondert die nachgeschaltete Nervenzelle eine cannabisähnliche Substanz ab, wird diese von den Astrozyten über sogenannte CB1-Rezeptoren wahrgenommen und an die vorgeschaltete Zelle weitergeleitet.

Dieser Zusammenhang könnte eine Erklärung dafür sein, weshalb Cannabis in gewissen Fällen erfolgreich als Schmerzmittel eingesetzt wird. Durch ein gezieltes Aktivieren der an diesen Synapsen sitzenden Astrozyten besteht nun die Hoffnung, Schmerzpatienten gezielt helfen zu können.

Literatur: Min R, Nevian T (2012) Astrocyte signaling controls spike timing-dependent depression at neocortical synapses. Nat Neurosci. Epub ahead of print, DOI: 10.1038/nn.3075

Quelle: Universität Bern, http://www.unibe.ch 